\title{
Impact of nuclear data on sodium-cooled fast reactor calculations
}

\author{
Alexander Aures ${ }^{1, a}$, Friederike Bostelmann ${ }^{1}$, Winfried Zwermann ${ }^{1}$, and Kiril Velkov ${ }^{1}$ \\ ${ }^{1}$ Gesellschaft für Anlagen- und Reaktorsicherheit (GRS) gGmbH, Boltzmannstr. 14,85748 Garching, Germany
}

\begin{abstract}
Neutron transport and depletion calculations are performed in combination with various nuclear data libraries in order to assess the impact of nuclear data on safety-relevant parameters of sodium-cooled fast reactors. These calculations are supplemented by systematic uncertainty analyses with respect to nuclear data. Analysed quantities are the multiplication factor and nuclide densities as a function of burn-up and the Doppler and Na-void reactivity coefficients at begin of cycle. While ENDF/B-VII.0 / -VII.1 yield rather consistent results, larger discrepancies are observed between the JEFF libraries. While the newest evaluation, JEFF-3.2, agrees with the ENDF/B-VII libraries, the JEFF-3.1.2 library yields significant larger multiplication factors.
\end{abstract}

\section{Introduction}

Among the envisaged Gen-IV reactor concepts are the sodium-cooled fast reactors (SFR). However, there is not much operation experience with these concepts and only very few data is publicly available. For a reliable prediction of safety-relevant parameters, it is necessary to correctly assess the applicability and quality of simulation tools and nuclear data libraries for the analyses of these systems. On the basis of the SFR-FT benchmark specification [1], the OECD/NEA Working Group SFR-FT [2] performed a parametric study of various SFR concepts: large size cores with oxide and carbide fuel and medium size cores with oxide and metallic fuel. The present contribution focuses on the large oxide core (MOX-3600) and the medium size metallic core (MET-1000). The objective of this study is to assess the impact of nuclear data on safety-relevant parameters such as the multiplication factor, nuclide densities, and the Doppler and Na-void reactivity coefficients. Neutron transport and depletion calculations with different nuclear data libraries are performed, and systematic uncertainty analyses with respect to nuclear data are carried out.

\section{Models and calculation methods}

For both cores considered, a model of a sub-assembly is built that corresponds to the lowest axial section of a fuel assembly of the inner core region. Detailed data of the geometry and the material is provided in the SFR-FT benchmark specification [1]. The coolant is sodium and the fuel consists mainly of U-238 and Plutonium and corresponds to the equilibrium state. The entire inventory of

\footnotetext{
${ }^{a}$ Corresponding author: alexander.aures@grs.de
} 
fission products is represented by a reactivity equivalent part of the molybdenum inventory. The fuel assemblies of the two core concepts are hexagonal and consist of 271 fuel pins.

\subsection{Calculation tools and nuclear data libraries}

The calculations in this study are performed with various neutron transport and depletion codes, and wherever possible - with different nuclear data libraries. The TRITON/NEWT and the TRITON/KENO sequence of the SCALE 6.1 code system [3] are applied with the 238-group ENDF/B-VII.0 library. Continuous-energy Monte Carlo calculations are undertaken with MCNP-6 [4] and Serpent 2 (2.1.24) [5] along with the nuclear data libraries ENDF/B-VII.1 (provided with MCNP6), ENDF/B-VII.0 and JEFF-3.1.1 (both provided with Serpent), and JEFF-3.1.2 and JEFF-3.2 (provided by OECD/NEA data bank).

Systematic uncertainty analysis with respect to uncertainties in the nuclear data is performed with the sampling-based XSUSA methodology (Cross Section Uncertainty and Sensitivity Analysis) and with the perturbation theory-based TSUNAMI module of the SCALE 6.1 code system. Details of both are given in Ref. [3,6].

\section{Results}

\subsection{Depletion calculations}

Depletion calculations are carried out for a sub-assembly of both the large oxide core (MOX-3600) and the ABR metallic core (MET-1000). The results are obtained by various stochastic and deterministic neutron transport and depletion codes, and by the application of different nuclear data libraries either in continuous-energy or in multi-group representation.

The fuel assembly of the large oxide core is depleted for 410 days with a thermal power of 50.4 $\mathrm{MW} / \mathrm{t}$ HM. Figure 1 shows the multiplication factor as a function of burn-up.

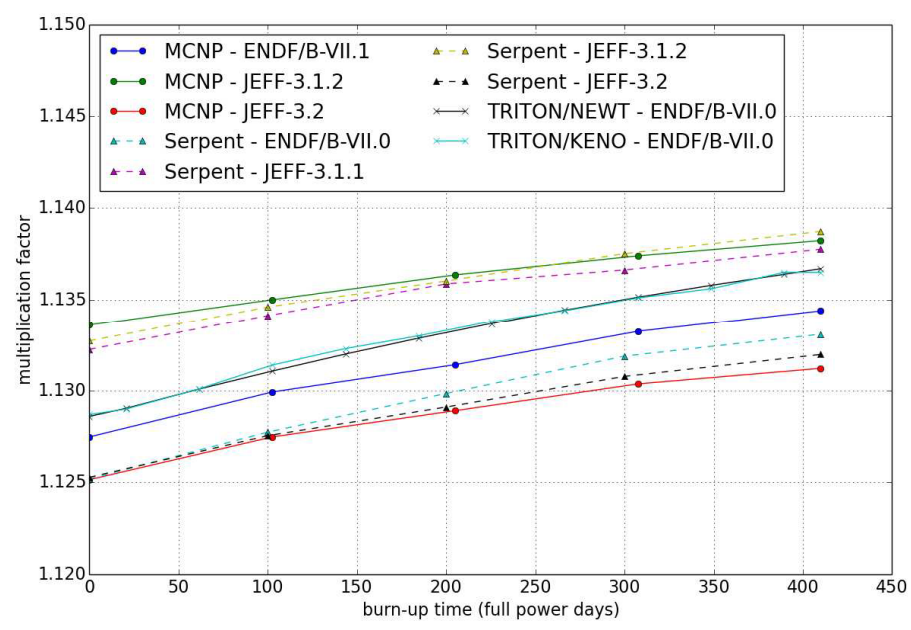

Figure 1. Multiplication factor as a function of burn-up from various neutron transport and depletion codes and different nuclear data libraries of a sub-assembly of the large oxide core.

The multiplication factors calculated by MCNP with JEFF-3.1.2 and ENDF/B-VII.1 shows a discrepancy of about $480 \mathrm{pcm}$ at BOC (begin of cycle) and $300 \mathrm{pcm}$ at EOC (end of cycle). This is in qualitative agreement with the results of other benchmark participants who obtained differences with 
the same sign and similar magnitude for whole core calculations when using ENDF/B-VII and JEFF3.1 data [2]. Applying the newest version of the JEFF library, JEFF-3.2, differences of $-184 \mathrm{pcm}$ at BOC and of $-247 \mathrm{pcm}$ at EOC to ENDF/B-VII.1 are obtained. Between JEFF-3.1.2 and JEFF-3.2, the differences are significant large, $-641 \mathrm{pcm}$ at BOC and $-514 \mathrm{pcm}$ at EOC. Similar results are obtained with Serpent. In case of JEFF-3.2, the multiplication factors calculated with Serpent and MCNP are consistent at BOC. However, with increasing burn-up, Serpent yields slightly larger multiplication factors. The results of the TRITON/NEWT and the TRITON/KENO sequences with application of the 238-group ENDF/B-VII.0 library are virtually identical. This is expected, since both codes use the same nuclear data library.

Moreover, the nuclide densities of several actinides obtained from the depletion calculations of the large oxide core are analysed at EOC. Figure 2 shows the main findings, namely the maximum relative deviations from the mean values of the nuclide densities.

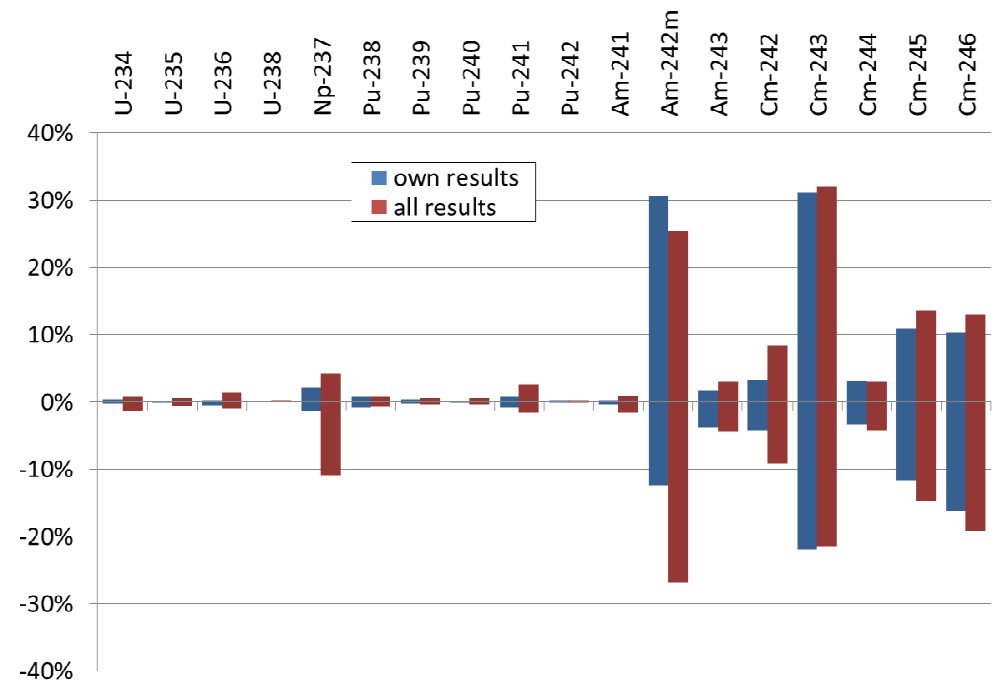

Figure 2. Range of results for actinide inventories at the end of the burn-up cycle obtained from own calculations only, and from own calculations and all available benchmark results.

The results denoted by own results are determined from the own TRITON/NEWT, MCNP (ENDF/BVII.1, JEFF-3.1.2) and Serpent (ENDF/B-VII.0, JEFF-3.1.1 / -3.1.2 / -3.2) calculations. TRITON/KENO results are excluded from the evaluation because they are almost identical to the TRITON/NEWT results. Additionally, the results are supplemented by corresponding values obtained from the results of the benchmark contributions (denoted by all results). While the bandwidths of the results are small for most actinides, in particular $\mathrm{Pu}-239$, they exhibit large values for $\mathrm{Np}-237$ and some isotopes of the Minor Actinides Am and Cm.

The sub-assembly of the ABR metallic core is depleted for 328.5 days with a thermal power of 75.4 $\mathrm{MW} / \mathrm{t} \mathrm{HM}$. Figure 3 shows the multiplication factors obtained with the various code systems and nuclear data libraries as a function of burn-up. Similar to the results of the large oxide core, a large discrepancy is observed between the multiplication factors obtained by MCNP with JEFF-3.1.2 and JEFF-3.2. The difference is about $850 \mathrm{pcm}$ at BOC and $800 \mathrm{pcm}$ at EOC. The difference between JEFF-3.2 and ENDF/B-VII.1 is about $200 \mathrm{pcm}$ at BOC and $20 \mathrm{pcm}$ at EOC. The multiplication factors calculated by the TRITON/NEWT sequence using the 238-group ENDF/B-VII.0 library are smaller than the continuous-energy Monte Carlo results. 


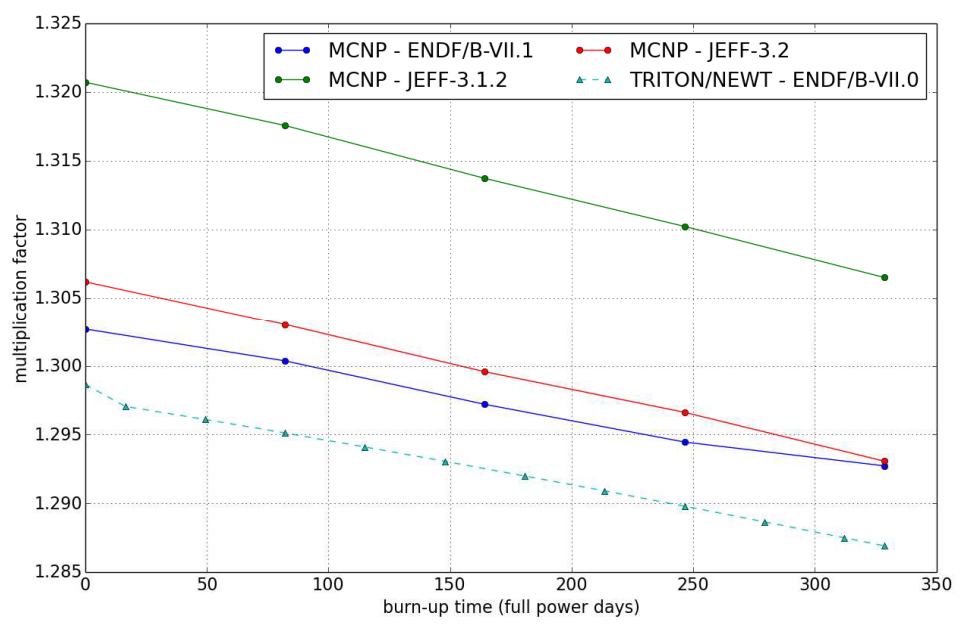

Figure 3. Multiplication factor as a function of burn-up from various neutron transport and depletion codes and different nuclear data libraries of a sub-assembly of the ABR metallic core.

\subsection{Safety-relevant parameters and their uncertainty with respect to nuclear data}

Safety-relevant parameters and their uncertainties are determined for the large oxide core and the ABR metallic core at BOC. For this, the temperature of all materials is set to $600 \mathrm{~K}$. For the assessment of the Doppler reactivity, the temperature is changed to $1200 \mathrm{~K}$, and for the sodium void reactivity, the sodium density is changed to $1 \%$ of the nominal density. Results of the safety-relevant parameters calculated with MCNP and different nuclear data libraries are presented in the Tables 1-2.

Table 1: MCNP results for the large oxide core fuel assembly at BOC.

\begin{tabular}{|l|l|l|l|l|}
\hline & ENDF/B-VII.0 & ENDF/B-VII.1 & JEFF-3.1.2 & JEFF-3.2 \\
\hline k-eff nominal & 1.13712 & 1.13901 & 1.14505 & 1.13677 \\
\hline k-eff high fuel temp. & 1.12823 & 1.13016 & 1.13620 & 1.12804 \\
\hline k-eff Na void & 1.17258 & 1.17481 & 1.18222 & 1.17361 \\
\hline Doppler reactivity $(\mathrm{pcm})$ & -693 & -688 & -680 & -681 \\
\hline Na void reactivity $(\mathrm{pcm})$ & 2659 & 2675 & 2746 & 2761 \\
\hline
\end{tabular}

Table 2: MCNP results for the ABR metallic core fuel assembly at BOC.

\begin{tabular}{|l|l|l|l|l|}
\hline & ENDF/B-VII.0 & ENDF/B-VII.1 & JEFF-3.1.2 & JEFF-3.2 \\
\hline k-eff nominal & 1.30246 & 1.30418 & 1.32281 & 1.30791 \\
\hline k-eff high fuel temp. & 1.29839 & 1.30020 & 1.31883 & 1.30399 \\
\hline k-eff Na void & 1.40546 & 1.40718 & 1.43291 & 1.41628 \\
\hline Doppler reactivity $(\mathrm{pcm})$ & -241 & -235 & -228 & -230 \\
\hline Na void reactivity $(\mathrm{pcm})$ & 5627 & 5612 & 5809 & 5850 \\
\hline
\end{tabular}

The differences in the Doppler reactivity are moderate for both fuel assemblies. However, significant differences can be observed between the multiplication factors and also for the sodium void reactivities. While ENDF/B-VII.0 and ENDF/B-VII.1 yield rather consistent results with a small and almost constant deviation in the multiplication factors for both cases, the deviations concerning the JEFF libraries are much larger. JEFF-3.1.2 yields substantially larger multiplication factors than the other libraries, in particular for the metallic core. 
Figure 4 shows results from the systematic uncertainty analyses with respect to nuclear data. On the left, uncertainty values of the multiplication factors, the Doppler and the Na-void reactivity are plotted for both systems for BOC. On the right, uncertainty values of the multiplication factor at BOC / EOC and of the actinide nuclide densities at EOC of the large oxide core (MOX-3600) are shown. The uncertainty of the multiplication factors is significantly larger than those found for light water reactor systems. The uncertainty of the nuclide densities is moderate compared to the bandwidths of the results from the various depletion sequences.
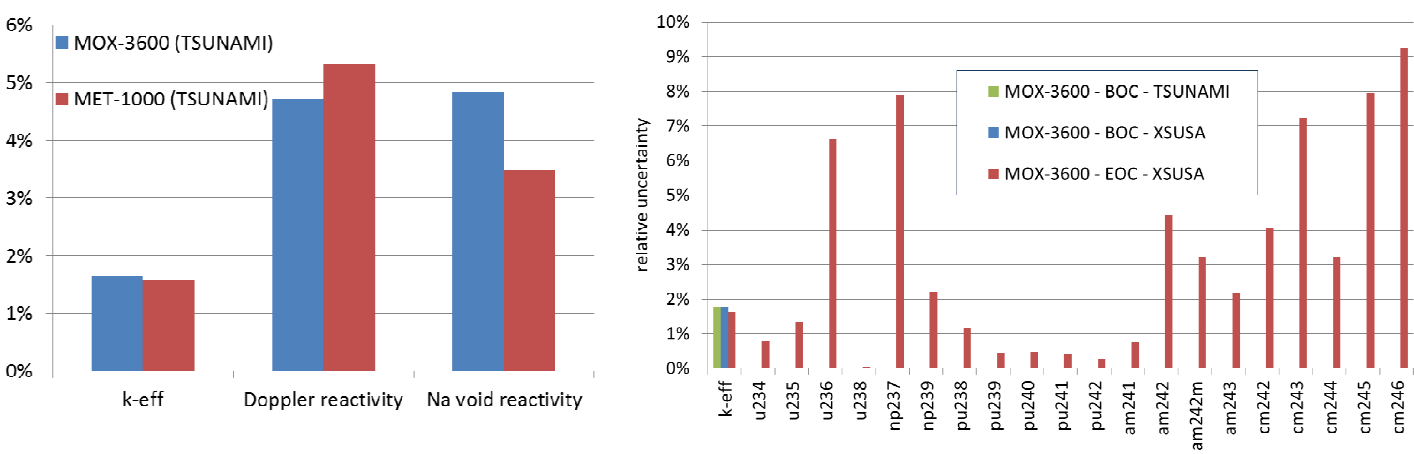

Figure 4. Uncertainties $(1 \sigma)$ of the multipl. factor, Doppler and Na-void reactivity for both systems at BOC (left), and of the multipl. factor and nuclide densities for the large oxide core at BOC / EOC (right).

\section{Summary}

In this study, the impact of nuclear data on safety-relevant parameters such as multiplication factor, nuclide densities, the Doppler reactivity coefficient and the Na-void reactivity coefficient is studied. Neutron transport and depletion calculations as well as systematic uncertainty analyses are performed for an axial section of a fuel assembly of both the large oxide core and the ABR metallic core. The application of different nuclear data libraries leads to large discrepancies in the multiplication factors as a function of burn-up for both fuel assemblies. The bandwidths of the nuclide densities of the actinides obtained from the various depletion sequences are moderate. Only for Np-237 and for several $\mathrm{Am}$ and $\mathrm{Cm}$ isotopes, larger bandwidths are observed. The analysis of the safety-parameters showed that the application of different nuclear data libraries results in significant discrepancies for the multiplication factors and sodium void reactivities. The uncertainty of the multiplication factor determined by the systematic uncertainty analysis turned out to be significantly larger than for LWR systems.

\section{Acknowledgments}

This work is supported by the German Federal Ministry for Economic Affairs and Energy.

\section{References}

1. D. Blanchet, L. Buiron, N. E. Stauff, T. K. Kim, T. Taiwo, AEN-WPRS Sodium Fast Reactor Core Definitions (Version 1.2 - September 19th), 2011, available at OECD/NEA Data Bank.

2. Sodium Fast Reactor Core Feed-back and Transient Response Task Force (SFR-FT Task Force), https://www.oecd-nea.org/download/wprs-sfr/index.html (2014)

3. SCALE: A Comprehensive Modeling and Simulation Suite for Nuclear Safety Analysis and Design, Version 6.1, ORNL/TM-2005/39, Oak Ridge National Laboratory, Oak Ridge, Tennessee, June 2011. Available from Radiation Safety Information Computational Center at Oak Ridge National Laboratory as CCC-785. 
4. J.T. Goorley et al., Initial MCNP6 Release Overview - MCNP6 version 1.0, Los Alamos National Laboratory, LA-UR-13-22934 (2013).

5. J. Leppänen, M. Pusa, T. Vittanen, V. Valtavirta, T. Kaltiaisenaho, The Serpent Monte Carlo code: Status, development and applications in 2013, Ann. Nuc. Energy 82, 142-150, (2015)

6. W. Zwermann, A. Aures, L. Gallner, V. Hannstein, B. Krzykacz-Hausmann, K. Velkov, J.S. Martinez, Nuclear Data Uncertainty and Sensitivity Analysis with XSUSA for Fuel Assembly Depletion Calculations, Nucl. Eng. and Tech. 46, No. 3, (2014) 